\section{Tribune du président de la SSDV Forum des Präsidenten der SGDV}

\author{
Mesdames, Messieurs, chers Collègues
}

Tout d'abord, une très bonne nouvelle puisque le Prof. JeanHilaire Saurat a été élu, lors du dernier congrès à Munich, pour être le futur Président de l'EADV. Nous le félicitons pour cette nouvelle tâche et lui souhaitons plein succès dans l'exercice de sa mission.

Ce numéro est spécialement constitué en vue de l'Assemblée générale extraordinaire qui se tiendra le 7 février 2002 à Berne.

Lors de la dernière Assemblée générale de notre Société à Genève, la majorité des personnes présentes a décidé de proposer une Assemblée générale extraordinaire vu l'importance du sujet du futur de notre Société.

Certains membres avaient l'impression que le Comité voulait retenir des informations, mais je peux vous confirmer que ce n'était pas du tout notre intention: nous ne voulions pas surprendre qui que ce soit. J'ai confirmé ceci à la fin du Congrès à Genève et j'aimerais reprendre la situation dans laquelle se trouvait le Comité. Le 16 mai, le Comité de la SSDV s'est réuni pour sa séance régulière et a proposé comme futur candidat à la présidence le Dr F. Gueissaz. M. Gueissaz nous annonce un projet de ses idées et comment il voit le futur de notre Société. Le 19 juillet, le Bureau s'étant réuni, le Dr Gueissaz présente son projet qui devrait être présenté au Comité le 26 septembre 2001: Le Comité s'est donc prononcé dessus et à l'unanimité a trouvé qu'il fallait le présenter à l'Assemblée générale. Toutefois, ce point n'étant préalablement pas prévu avec certitude ne pouvait figurer à l'ordre du jour de l'Assemblée générale du programme publiée dans Dermatologica Helvetica. De plus, le comité a trouvé qu'il serait mieux de publier le projet du Dr Gueissaz dans un futur numéro de Dermatologica Helvetica afin de permettre à tous les membres de s'exprimer sur ce sujet puisque, par expérience, nous savons que tout le monde n'assiste pas à l'Assemblée générale.

Puisque l'Assemblée générale extraordinaire a maintenant été décidée (voir ordre du jour ci-joint), il me paraît salutaire que nous puissions nous exprimer sur le projet du Dr Gueissaz et établir un dialogue. Par la même occasion, je vous saurais gré de bien vouloir formuler préalablement votre question sur la feuille ci-jointe (p. 27) qui nous permettra de structurer le débat (proposition du Dr P. Baumann).

De plus il est aussi question d'une éventuelle résiliation de la FMS. A ce sujet, il m'apparaît important d'attirer votre attention sur le fait que notre Société est également - et entre autres - une société chirurgicale et qu'il est nécessaire de bien réfléchir sur ce point puisque nous ne savons pas si nous regretterons d'avoir quitté la FMS.

Dans un des prochains numéros, nous projetons de publier les nouvelles propositions pour la révision des statuts.

En vous invitant à venir nombreux à cette Assemblée générale extraordinaire où sera discuté le futur de votre Société, je vous remercie d'ores et déjà de votre participation active et je vous transmets, Mesdames, Messieurs, chers Collègues, mes meilleures salutations.

Prof. R.G. Panizzon

\section{Liebe Kolleginnen und Kollegen}

Vorerst eine erfreuliche Nachricht: Prof. Jean-Hilaire Saurat ist anlässlich des Kongresses in München zum zukünftigen Präsidenten der EADV gewählt worden. Herzlichen Glückwunsch! Wir wünschen ihm für seine wichtige Aufgabe viel Erfolg.

Diese Nummer der «Dermatologica Helvetica» ist speziell für die ausserordentliche Generalversammlung, die am 7. Februar 2002 in Bern stattfindet, gedacht.

Anlässlich der letzten Generalversammlung unserer Gesellschaft in Genf hat eine Mehrheit der anwesenden Mitglieder beschlossen, eine ausserordentliche Generalversammlung einzuberufen, um über die Zukunft unserer Gesellschaft zu debattieren.

Einige Mitglieder hatten den Eindruck, dass der Vorstand gewisse Informationen zurückbehalten und die Mitglieder nicht vollständig informiert hat. Ich kann Ihnen versichern, dass dies absolut nicht unsere Absicht war, wir wollten niemanden überraschen. Wie ich anlässlich meiner Schlussrede in Genf ausgeführt habe, möchte ich die Situation des Vorstandes nochmals darlegen: Am 16. Mai anlässlich der Vorstandssitzung wurde Dr. F. Gueissaz als Kandidat für das Präsidentenamt vorgeschlagen. Dr. Gueissaz hat daraufhin bekanntgegeben, dass er seine Ideen und die Zukunftsvision unserer Gesellschaft vorbereitet. Am 19. Juli anlässlich einer Ausschuss-Sitzung hat Dr. Gueissaz sein Projekt vorgelegt und dies dann auch am 26. September im Vorstand vorgetragen. Der Vorstand hat einstimmig beschlossen, dass dieses Projekt anlässlich der Generalversammlung vorgestellt werden sollte. Da dieser Punkt nicht definitiv für die Traktandenliste der Generalversammlung vorgesehen war, ist dieser auch nicht in der «Dermatologica Helvetica» publiziert worden. Im übrigen war es auch die Ansicht des Vorstandes, das Projekt von Dr. Gueissaz in einer Nummer der «Dermatologica Helvetica» zu publizieren, damit sämtliche Mitglieder sich dazu äussern können, denn erfahrungsgemäss nehmen ja nicht alle Mitglieder an der Generalversammlung teil.

Nun, da die ausserordentliche Generalversammlung beschlossene Sache ist (s. Traktandenliste in diesem Heft), finde ich, dass es gut ist, sich zu diesem Projekt äussern zu können. Bei dieser Gelegenheit möchte ich Sie aber bitten, mir vorgängig Ihre Fragen schriftlich auf dem Fragebogen (p. 27) zu formulieren, damit wir eine geordnete Debatte durchführen können (Vorschlag von Dr. P. Baumann).

Es besteht ferner ein Vorschlag betreffend Austritt aus der FMS. Diesbezüglich glaube ich, dass es wichtig ist, Sie darauf aufmerksam zu machen, dass unsere Gesellschaft auch eine chirurgisch tätige Gesellschaft ist, und es deshalb wichtig ist, darüber nachzudenken, ob wir zu einem späteren Zeitpunkt einen Austritt bereuen könnten.

In einer der nächsten Nummern von «Dermatologica Helvetica» möchten wir auch die Vorschläge zu Statutenänderungen publizieren.

Nun möchte ich Sie bitten, zahlreich zur ausserordentlichen Generalversammlung zu erscheinen, um über die Zukunft unserer Gesellschaft zu diskutieren, und bedanke mich schon jetzt für Ihre aktive Teilnahme.

Mit freundlichen Grüssen Prof. R.G. Panizzon 